\title{
¿Cuál es el algoritmo diagnóstico más apropiado para el cribado del cáncer de próstata?
}

\author{
C. Allepuz Losa, Á. Borque Fernando, L.A. Rioja Sanz
}

Servicio de Urología. Hospital Universitario Miguel Server. Zaragoza.

Actas Urol Esp 2005; 29 (5): 465-472

\section{RESUMEN \\ ¿CUÁl ES EL ALGORITMO DIAGNÓSTICO MÁS APROPIADO PARA EL CRIBADO DEL CÁNCER DE PRÓSTATA?}

Desde la generalización del uso del PSA para la detección del cáncer de próstata en los últimos años de la década de los 80, el diagnóstico del cáncer de próstata se ha incrementado de forma notable. Aunque hoy día existe evidencia indirecta que haría pensar en un efecto beneficioso del cribado, no hay datos que justifiquen el cribado con PSA en la población general. Así mismo, existe controversia sobre cual sería el algoritmo más apropiado en el caso de que el cribado se realice. Por todo ello, nuestro objetivo ha sido realizar una revisión de la literatura reflexionando en base a nuestra experiencia para intentar definir cual sería el mejor algoritmo para el cribado del cáncer de próstata. Para ello, hemos realizado una búsqueda en Medline cruzando los términos biopsia de próstata, cribado o screening, algoritmos, prostatectomía radical, PSA y cáncer de próstata.

Después del análisis de la literatura, pensamos que no existe un algoritmo "definitivo" debido al carácter dinámico de los mismos por la rápida aparición e incorporación de nuevos avances técnicos y biológicos, pero parecería que hoy por hoy, sin dejar de incorporar el tacto rectal, se debería dar más valor a los factores personales de riesgo, y entre ellos el PSA a edades inferiores a 50 años realizando un seguimiento individualizado en función de estos factores. Los algoritmos aplicados a una población deberian de ser validados previamente en dicha población.

Palabras Clave: Cáncer de próstata. Screening. Biopsia de próstata. PSA. Diagnóstico precoz.

\section{ABSTRACT}

\section{¿WHICH IS THE MOST APPROPRIATE DIAGNOSTIC ALGORITHM FOR PROSTATE CANCER SCREENING?}

Since the use of PSA to detect prostate cancer was generalised in the late 1980's, prostate cancer diagnosis has increased considerably. Although there is now indirect evidence pointing to the beneficial effect of screening, there are no data justifying PSA screening in the general population. There is also a controversy concerning the most appropriate algorithm, should screening be performed. Therefore, our aim was to review the literature and, based on our experience, attempt to define the best algorithm for prostate cancer screening. We have made a search on Medline using the following terms: prostate biopsy, screening, algorithms, radical prostatectomy, PSA and prostate cancer.

After analysing the literature, we can confirm that there is no "definitive" algorithm, due to the rapid appearance and use of new technical and biological breakthroughs, although it appears that at this time, without ceasing to include a rectal examination, more value should be given to personal risk factors, including PSA, at ages under 50, with individual monitoring based on these factors. The algorithms applied to a population have first to be validated for the population concerned.

Keywords: Prostate cancer. Screening. Prostate biopsy. PSA. Early diagnosis. 
$\mathrm{D}$ esde la generalización del uso del PSA para la detección del cáncer de próstata en los últimos años de la década de los 80 , el diagnóstico del cáncer de próstata se ha incrementado de forma notable. Este incremento, ha traído consigo un encendido debate sobre la conveniencia de realizar cribado con PSA del cáncer de próstata ${ }^{1}$.

Existe evidencia indirecta que haría pensar en un efecto beneficioso del cribado. Después de la incorporación del PSA, el estadio del tumor al diagnóstico ha cambiado, hallándose más casos órgano-confinados y menos metástasis a distan$\mathrm{cia}^{2}$. En la época pre-PSA sólo el $33 \%$ de los tumores detectados mediante tacto rectal eran patológicamente órgano-confinados, frente al 62$71 \%$ en la actualidad ${ }^{3}$. Además la diferenciación tumoral también ha cambiado, incrementándose el número de casos moderadamente diferencia$\operatorname{dos}^{2}$ y disminuyendo en algunos estudios en un $33 \%$ los mal diferenciados ${ }^{4}$. Nuestro programa de diagnóstico precoz ${ }^{5}$, basado en la utilización del PSA y tacto rectal y realizado en población remitida desde una consulta médica externa, también ha confirmado estos hechos, de manera que en 1991 la mediana de PSA de los pacientes diagnosticados de cáncer de próstata era de 32,42 $\mathrm{ng} / \mathrm{ml}$. frente a $16,25 \mathrm{ng} / \mathrm{ml}$ en 1995 y $7,6 \mathrm{ng} / \mathrm{ml}$ en el año 2000. Además el $48 \%$ de los pacientes en 1991 con edad de 70 años o menor a los que se les practicó una prostatectomía radical derivada del diagnóstico de cáncer de próstata en este programa tenían el tumor órgano confinado, frente al $62,5 \%$ en 1995, y el $77 \%$ en el año 2000 y lo que es más demostrativo, el volumen tumoral hallado en la pieza de prostatectomía descendió de 3,87 cc de mediana a 1,35 cc en 1995 y a 1,1 cc en el año $2000^{1}$.

En EEUU la mortalidad por cáncer de próstata creció, hasta 1991, fecha en la que empezó a descender llegando a una tasa anual de 1,9\% en 1995, siendo asumido por una gran parte de los autores, que el origen sería la combinación de un cribado y tratamientos agresivos ${ }^{6}$. En Europa los datos más sugestivos son los provenientes de un estudio realizado en el Tirol (Austria), en donde el cribado con tacto rectal y PSA comenzó en 1993, descendiendo desde entonces un $42 \%$, a diferencia de otros lugares de Austria en los que no se llevó a cabo el cribado? .
No obstante, no existen hoy día datos que justifiquen el cribado con PSA en la población general. Cabe esperar que datos a este respecto deriven de los resultados de dos ensayos clínicos, el Prostate, Lung, Colorectum and Ovary(PCLO) Sreening Trial que se desarrolla en USA y el European Randomised Screening Trial for Prostate Cancer(ERSPC), cuyos resultados definitivos se esperan en torno al $2008^{8}$.

A nivel Español también el cribado poblacional se ha desestimado en base a los resultados obtenidos por Martín y cols. ${ }^{9}$, habiéndose propugnado sin embargo el cribado oportunístico ${ }^{10} \mathrm{y}$ en pacientes con sintomatología miccional ${ }^{5}$.

Pero no sólo existe ausencia de certeza en cuanto a si el cribado es capaz de mejorar la supervivencia y disminuir la mortalidad, sino que además, cuando este cribado se realiza, en la actualidad, existe una gran controversia en cuanto a cual sería el algoritmo más apropiado. Por todo ello, nuestro objetivo ha sido realizar una revisión de la literatura reflexionando en base a nuestra experiencia para intentar definir cual sería el mejor algoritmo para el cribado del cáncer de próstata. Para lograr nuestro objetivo, hemos realizado una búsqueda en Medline cruzando los términos biopsia de próstata, cribado o screening, algoritmos, prostatectomía radical, PSA y cáncer de próstata

\section{¿CUÁNDO EMPEZAR EL CRIBADO?}

A la hora de considerar cuando debemos de empezar a realizar el cribado, las consideraciones serán diferentes si hablamos de la forma hereditaria o de la forma esporádica de cáncer de próstata.

En la actualidad, es un hecho contrastado que los antecedentes familiares suponen un importante factor de riesgo para padecer cáncer de prósta$\mathrm{ta}^{11}$. No obstante, como los genes responsables todavía no han sido clonados, la definición de cáncer de próstata hereditario se basa sólo en el árbol genealógico. La definición más aceptada incluye núcleos familiares con 3 casos o más de cáncer de próstata, familias con cáncer de próstata en 3 generaciones de la línea materna o paterna, y familias con dos varones diagnosticados de la enfermedad antes de los 55 años $^{12}$. No obstante dada la dificultad de identificar portadores femeninos de mutaciones, la utilidad del árbol genealógico para detectar familias susceptibles de heredar cáncer de próstata es muy limitada ${ }^{11}$. 
El impacto de la historia familiar en la probabilidad de sufrir cáncer de próstata supone un incremento de riesgo relativo entre 1,5 y 5 veces en función de un mayor número de parientes varones afectos o un parentesco más próximo debiendo remarcarse que esta probabilidad se incrementa notablemente en aquellos casos en los que el familiar padeció la enfermedad en edades más tempranas ${ }^{11}$. Grönberg y cols. ${ }^{13}$ encuentran una probabilidad de desarrollar cáncer de próstata clínico antes de los 70 años del 15\% en pacientes con 2 o más familiares cercanos con antecedentes de la enfermedad, probabilidad que se incrementa al 30-45\% si el familiar fue diagnosticado antes de los 70 años. Se considera que entre el 5-10\% de los cánceres de próstata están causados por mutaciones heredadas con alta penetrancia $^{12,14}$, porcentaje que se incrementa al $33 \%$ de los casos diagnosticados antes de los 60 años y a más del 40\% de los diagnosticados antes de los 55 años ${ }^{14,15}$.

Desde un punto de vista clínico la forma hereditaria aparece 6-7 años antes que la esporádica, lo que incrementaría la probabilidad de morir por cáncer de próstata si este no fuera diagnosticado de forma precoz. La mayoría de los estudios muestran similares características entre los 2 $\operatorname{tipos}^{11}$, siendo además los hereditarios en su mayoría tumores importantes y con buenos factores pronósticos ${ }^{16}$.

Por todo ello una gran mayoría de los autores recomiendan comenzar el cribado del cáncer de próstata en pacientes con historia familiar, a partir de los 40-45 años, particularmente si tienen 2 ó más familiares afectados con la enfermedad ${ }^{11,16,17}$.

Distinto es el caso de la forma esporádica del cáncer de próstata. En estos casos cuando se propugna una postura activa, se ha recomendado comenzar el cribado a partir de los 50 años, basándose en el incremento del cáncer de próstata a partir de esta edad ${ }^{17-19}$. Si bien esta postura ha sido ampliamente aceptada y puede ser válida, se han postulado también otras posturas que conllevan la realización de test de forma rutinaria en pacientes más jóvenes ${ }^{18}$, en este sentido Ross y cols. ${ }^{20}$ comparando estrategias de cribado valorando resultados de salud(muertes por cáncer de próstata evitadas y años de vida ahorrados / persona) y utilización de recursos(test de PSA y biop- sias de próstata) encuentran que la estrategia más costo-eficiente conllevaría comenzar a los 40 años las determinaciones de PSA estableciendo una línea de base, y haciendo posteriormente nuevas determinaciones a los 45, 50 años y luego de forma bianual.

\section{¿QUÉ MÉTODOS UTILIZAR PARA INDICAR LA} PRIMERA BIOPSIA?

Las principales pruebas de cribado del cáncer de próstata son el tacto rectal, la ecografía transrectal y la determinación serológica de marcadores tumorales como el antígeno prostático específico.

\section{Ecografia transrectal}

En la actualidad se reconoce que la ecografia transrectal tienen una escasa sensibilidad especificidad y valor predictivo positivo, y su utilización conllevaría un una gran cantidad de recursos ${ }^{21}$. No obstante si bien el uso de la ecografía transrectal en escala de grises ha quedado casi limitada a la determinación del volumen prostático y como guía de biopsia, se están desarrollando mejoras que pueden en un futuro volver a colocar a esta técnica como elegible de primera intención en el diagnóstico del cáncer de próstata ${ }^{22}$.

\section{Tacto rectal}

El tacto rectal tiene una baja sensibilidad, habiéndose recogido, que del 25 al 50\% de los cánceres de próstata son diagnosticados con tacto rectal no sospechoso ${ }^{21,23}$. Así mismo se le ha atribuido un valor predictivo positivo variable entre el 5 y el 83\%, variabilidad que podría estar mediatizada por el PSA, raza, antecedentes familiares, edad y nivel de $\mathrm{PSA}^{11,21,23}$. Por todo ello, aunque la utilidad del tacto rectal en el cribado del cáncer de próstata, sigue siendo defendida por gran parte de los autores ${ }^{24,25}$, ha sido puesta recientemente en entredicho.

Carvalhal y cols. ${ }^{25}$ encuentran en pacientes con PSA menor de $4 \mathrm{ng} / \mathrm{ml}$ que el valor predictivo positivo varía con el nivel de PSA de forma que sería de 5, 14 y 30\% para intervalos de PSA de 0 a $1.0,1.1$ a 2.5 y 2.6 a $4.0 \mathrm{ng} / \mathrm{ml}$. respectivamente. Todos los casos fueron clínicamente localizados y del 72\% quirúrgicamente estadiados el $82 \%$ fueron órgaconfinados y el 78\% fueron tumores moderadamente diferenciados. Además, 
este grupo también encuentra un $88,1 \%$ de enfermedad significativa en pacientes intervenidos con PSA entre 2,6 y 4 ng. $/ \mathrm{ml}^{26}$. En base a todos estos hallazgos estos autores recomienda la realización del tacto rectal junto con la determinación de PSA en pacientes sometidos inicialmente a cribado por cáncer de próstata.

Otros autores como Vis ${ }^{27,28}$ y Schröder ${ }^{29}$, con los datos de las bases de la ERSPC(European Randomized Study of Screening for Prostate Cancer), sección de Rótterdam, encuentran que el tacto rectal tiene un menor valor predictivo positivo que el descrito anteriormente y un mayor porcentaje de cánceres hallados que podrian definirse como potencialmente insignificantes o no buscados(Tabla 1), lo que les indujo a pensar que se podría prescindir de la realización del tacto rectal, si se bajaba el punto de corte de PSA considerado como patológico.

Para validar esta teoría, Schröder y cols. ${ }^{30}$ hicieron un estudio comparando dos algoritmos distintos, el inicial, en el que eran indicación de biopsia de próstata la existencia de un PSA mayor de 4 $\mathrm{ng} / \mathrm{ml}$, un tacto rectal sospechoso o lesiones sospechosas en la ecografia transrectal, con un algoritmo nuevo, en el que la indicación de biopsia se basaba sólo en la existencia de un PSA mayor de 3 $\mathrm{ng} / \mathrm{ml}$ La tasa de detección de cáncer fue muy similar(5,0 versus 4,7\%). El Valor Predictivo Positivo del nuevo algoritmo fue mayor que el del antiguo, $24,3 \%$ versus $18,2 \%$ respectivamente $(\mathrm{p}<0.001)$, siendo el número de biopsias necesario para diagnosticar un cáncer de 5,2 y 3,4 respecti-

\section{Tabla 1}

Valores predictivos positivos, porcentaje de enfermedad significativa ${ }^{27}$ y porcentaje de cánceres no buscados ${ }^{28}$ estratificados por distintos niveles de PSA en pacientes con PSA total menor de 4 ngr/ml Enfermedad significativa: tumor mayor de $0,5 \mathrm{ml} \mathrm{o}$ menor no órganoconfinado o con patrón de Gleason primario de 4 ó 5. Cánceres no buscados: tumores menores de 0,5 cc y/o cáncer en otra localización al sospechado por ecografía transrectal o tacto rectal.

\begin{tabular}{cccc}
\hline PSA (ng/ml) & VPP TR(+) & $\begin{array}{c}\text { Enfermedad } \\
\text { Significativa (\%) }\end{array}$ & $\begin{array}{c}\text { Cánceres no } \\
\text { buscados (\%) }\end{array}$ \\
\hline $0,0-1,0$ & 2,2 & 0,0 & 100 \\
$1,0-2,0$ & 8,5 & 26,3 & 36 \\
$2,0-2,9$ & 13,4 & 60,0 & 40 \\
$3,0-3,9$ & 25,4 & 67,5 & 20 \\
\hline
\end{tabular}

VPP TR(+): Valores predictivo positivo de un tacto rectal sospechoso de cáncer. vamente. Además, dentro de los pacientes a los que se les practicó una prostatectomía radical en el anterior algoritmo, se encontró una similar distribución en la suma del grado del Gleason, pero con una mayor proporción de enfermedad órganoconfinada. Los volúmenes tumorales fueron menores en pacientes con un PSA menor de $2.9 \mathrm{ng} / \mathrm{ml}$. y la proporción de enfermedad mínima en este grupo de pacientes fue del $50 \%$ comparado con el $28 \%$ hallado en pacientes con PSA entre 3,0 y 3,9\%. Estos autores concluyen en que el algoritmo basado en la determinación de PSA con un punto de corte de 3 $\mathrm{ng} / \mathrm{ml}$ como indicación de biopsia es beneficioso en una población de cribado poblacional, asumiendo el 16\% de los cánceres que no se hubieran diagnosticado en el intervalo de PSA de 1,0-2,9 ng/ml, si bien este beneficio sería menor en la practica clínica, en donde el tacto rectal se realiza habitualmente antes de conocer el PSA y por motivos muchas veces distintos al diagnóstico del cáncer de próstata.

Aún siendo los datos de Schröder y cols. ${ }^{30}$ muy valorables, y dignos de tenerlos en consideración, hay que tener en cuenta que la comparación de algoritmos no esta realizada en la misma muestra sino en poblaciones consecutivas y con 5 años de diferencia entre el inicio de la una y el final de la otra, pudiendo conllevar ambos aspectos importantes sesgos. Además los datos presentados en relación a las prostatectomías realizadas sólo se basan en el algoritmo clásico, no siendo realmente comparativos entre ambos algoritmos. Por último, un $16,1 \%$ de los pacientes a los que se indicó biopsia con el nuevo algoritmo no llegaron a realizársela, frente al 10,7\% en el anterior algoritmo, pudiendo esto también haber distorsionado en alguna medida los resultados.

En resumen, si bien, el omitir el tacto rectal en un programa de cribado poblacional podría llegar a ser una opción válida, hoy por hoy no esta generalizada, bien al contrario, la mayoría de los autores recomiendan la utilización de ambas pruebas, PSA y tacto rectal, de forma más determinante en la práctica clínica habitual.

\section{PSA}

Habitualmente se considera que el intervalo de normalidad del PSA total sérico oscila entre 0 y $4 \mathrm{ng} / \mathrm{ml}^{17}$. Con el punto de corte de $4 \mathrm{ng} / \mathrm{ml} \mathrm{el}$ 
PSA presenta una sensibilidad en torno al $80 \%^{21}$, habiéndose propuesto variar el punto de corte con el objeto de aumentar la sensibilidad.

Guizá una de las primeras iniciativas para optimizar el punto óptimo de corte del PSA viniera de la mano de Oesterling ${ }^{31}$, que lo estratificaba en función de distintos intervalos de edad del paciente, pretendiendo con ello aumentar la sensibilidad en jóvenes y la especificidad en pacientes mayores. Su uso no se ha generalizado debido a que aunque suponía un ahorro de un 15\% de biopsias, también suponía una pérdida de diagnósticos de cánceres órganoconfinados del $8 \%$ en pacientes entre 60 y 69 años y de un ahorro de un $44 \%$ de biopsias a costa de una pérdida de diagnósticos de cánceres órganoconfinados del $47 \%$ en pacientes mayores de 70 años $^{32}$.

Posteriormente se han recogido las propuestas de disminución de forma uniforme del punto de corte de PSA a 2,6 o $3 \mathrm{ng} / \mathrm{ml}$ basándose en que en el intervalo de PSA de 2,6 a $4 \mathrm{ng} / \mathrm{ml}$. se encuentran alrededor de un 20 a 30\% de cánceres $^{17,29,30,33}$. Debido a que esta disminución del punto de corte suponía un incremento del número de biopsias que se debían de realizar, el doble por cada cáncer curable ${ }^{18}$, con el objeto de aumentar la especificidad de la indicación de biopsia, se han utilizado distintas metodologias dentro de este intervalo de PSA de 2,6 a $4 \mathrm{ng} / \mathrm{ml}$, como el \%PSA libre, PSA densidad del adenoma y de la próstata total, con los que se podrían evitar el 17 al $29 \%$ de las biopsias con sensibilidades del 95\% ${ }^{34}$. Asî mismo, el PSA velocidad también podría ser útil, ya que valores de $0,1 \mathrm{ng} / \mathrm{ml} /$ año en pacientes con PSA total entre 2 y $4 \mathrm{ng} / \mathrm{ml}$ presentan una especificidad del $50 \%$ con una sensibilidad del $80 \%{ }^{35}$.

Por otra parte, Carter y cols. ${ }^{36}$ refiriéndose no ya a la detección global de cáncer sino a la de cáncer curable(tumor órganoconfinado o con penetración capsular con Gleason $<7$ y márgenes negativos), encuentra en 389 pacientes con cáncer de próstata no palpable tratados con cirugía radical y extraídos de la "Baltimore Longitudinal Study of Aging Database" un 94\%, 89\% y 70\% de cáncer curable en pacientes con PSA menor de 4 , entre 4 y 5 y mayor de $5 \mathrm{ng} / \mathrm{ml}$. respectivamente. Parece claro que no es asumible desear la detección de sólo un $70 \%$ de cáncer curable, por lo que el punto de corte de PSA de $4 \mathrm{ng} / \mathrm{ml}$ se muestra como razonable y válido, ya que mantiene en porcentajes altos la detección de enfermedad curable. Así mismo, Carter y cols. ${ }^{37}$, encuentra que la probabilidad de diagnosticar cáncer curable en pacientes sin enfermedad palpable varía menos de un $4 \%$ entre intervalos de PSA de 2,6-4 ng/ml y 4-6 ng/ml, y esto ocurre para todos los grupos de edad(40-50, 51-60 y 61-73 años), por lo que reitera su convencimiento de la inconveniencia de disminuir el punto de corte de PSA, y sí en todo caso disminuir la edad de inicio de estudio de los pacientes.

$\mathrm{Y}$ es en la disminución de la edad de comienzo de estudio, donde numerosos autores opinan que quizás lo más eficiente sería determinar el PSA basal a edades más tempranas, individualizando la conducta a seguir en función de éste. En este sentido Gann y cols. ${ }^{38}$ en pacientes de 63 años de media y basado en muestras de sange recogidas y congeladas 10 años antes refieren que los pacientes con niveles séricos de PSA entre 1,01 y 1,50 $\mathrm{ng} / \mathrm{ml}$ tenían un riesgo 2 veces mayor que los pacientes con PSA entre 0,0 y $1,0 \mathrm{ng} / \mathrm{ml}$ de ser diagnosticados de cáncer de próstata en ese periodo de tiempo, riesgo que se incrementaba a 5 veces para los pacientes con un PSA de 2,01 a $3 \mathrm{ng} / \mathrm{ml}$. Fang y cols. ${ }^{39}$ en pacientes de 50 a 59 años observa que aquellos que tienen un PSA menor de 0,7 $\mathrm{ng} / \mathrm{ml}$ tienen 3 veces menos riesgo de padecer cáncer de próstata en las 2 siguientes décadas en comparación con los que su PSA es mayor.

En resumen, y a la vista de lo expresado en la literatura hasta el momento, parecería que la conducta más eficiente sería la individualizada, en función del PSA inicial de base, conducta que sería más racional que disminuir el punto de corte de PSA.

\section{Modelos Predictivos}

Recientemente han proliferado la creación de modelos predictivos ya sea basados en regresión logística o redes neuronales ${ }^{40,41}$ para predecir el resultado de la primera biopsia en función de distintas variables de entrada. Creemos que si bien estos modelos pueden ser útiles, algunos están todavía en fase de desarrollo y otros, los más en fase de validación. Quizá la máxima utilidad provenga de aquellos que incorporen más y nuevas variables, alguna de ellas relacionadas con el PSA de base y el tiempo. 


\section{¿CUÁL ES EL MEJOR INTERVALO ENTRE TEST?}

$\mathrm{El}$ intervalo anual entre revisiones en pacientes con tacto rectal no sospechoso y PSA menor de $4 \mathrm{ng} / \mathrm{ml}$ ha sido el estándar hasta la fecha, pero puede que no sea precisa su realización con tanta frecuencia. En este sentido, ya hemos visto que se ha descrito una clara relación entre la cifra de PSA inicial y la probabilidad de desarrollar un cáncer de próstata ${ }^{36,38,39}$. A estos datos, se unen los presentados por Smith y cols. ${ }^{42}$ que encuentran como en pacientes mayores de 50 años y en un periodo de 4 años, sólo un $4 \%$ de seroconversión del PSA a valores mayores de 4 $\mathrm{ng} / \mathrm{ml}$ cuando se partía de un PSA inicial igual o inferior a $2,5 \mathrm{ng} / \mathrm{ml}$ frente al $48 \%$ de seroconversión cuando el PSA inicial era mayor de 2,5 $\mathrm{ng} / \mathrm{ml}$ Carter y cols. ${ }^{36}$ también observan como si partimos de un PSA inicial menor de $2 \mathrm{ng} / \mathrm{ml} \mathrm{la}$ probabilidad de conversión a PSA > $5 \mathrm{ng} / \mathrm{ml}$, (situación en la que la probabilidad de hallar cáncer no curable es mayor) es del $0 \%$ a 2 y 4 años, y la probabilidad de conversión a $4-5 \mathrm{ng} / \mathrm{ml}$ es de un $4 \%$ a 2 años, pero la probabilidad de conversión a PSA entre 4 y $5 \mathrm{ng} / \mathrm{ml}$ es de un $27 \%$ a los 2 años si el PSA inicial entre 2,1-3 ng/ml y de un $36 \%$ si el PSA inicial estaba entre 3.1 a $4 \mathrm{ng} / \mathrm{ml}$, concluyendo que un intervalo entre determinaciones de PSA de 2 años sería seguro en pacientes con un PSA inicial menor de $2 \mathrm{ng} / \mathrm{ml}$.

Así mismo, Leewangston y cols. ${ }^{43}$ describen en base a los datos de la Prostate Cancer Awareness Week que a 3 años la probabilidad de encontrar cáncer era mayor en función del PSA inicial en pacientes con PSA menor de 4, de modo que en aquellos con un PSA inicial de 2,1-4 ng/ml encontraron 3 y 6 veces más cánceres que en aquellos que presentaban inicialmente un PSA de 1,1-2 $\mathrm{ng} / \mathrm{ml}$ y 0-1 $\mathrm{ng} / \mathrm{ml}$ respectivamente.

En esta línea, recientemente Ross y cols. ${ }^{20}$ utilizando la simulación de Monte-Carlo basada en Modelos de Markov describe que el Algoritmo basado en la determinación de PSA más eficiente para el cribado del cáncer de próstata, era aquel en el que estableciendo como indicación de biopsia un PSA mayor de $4 \mathrm{ng} / \mathrm{ml}$, comenzara a los 40 años estableciendo una línea de base de PSA, y realizando posteriormente nuevas determinaciones a los 45 y 50 años en el caso de que el PSA se mantuviera en niveles menores a $2 \mathrm{ng} / \mathrm{ml}$ o de forma bianual en caso contrario, para continuar a partir de los 50 años de forma bianual.

Por tanto, parece que la conducta óptima para determinar los intervalos entre test sería aquella que tuviera en cuenta el PSA basal, individualizando en función de esta, los posteriores seguimientos, que en determinados casos parece claro que podrian ser superiores al clásico anual, sin perder seguridad en el diagnóstico.

\section{¿CUÁNDO TERMINAR?}

Conocidos los datos anteriores también podríamos inferir que en función de ciertos niveles de PSA la edad a la que deberemos interrumpir los test de detección de cáncer de próstata no será generalizada.

En la actualidad parece que existe acuerdo en relación a que en líneas generales la detección del cáncer de próstata debe interrumpirse en pacientes con una expectativa menor de 10 años, ya que en estos no existe un claro beneficio en cuanto a supervivencia y si puede haber un decremento en calidad de vida ${ }^{18}$.

Carter ${ }^{44}$ en un estudio en pacientes mayores de 60 años, evalúa la asociación entre la edad, la línea basal del PSA y la detección de cáncer de próstata, encontrando que ninguno de sus pacientes con edades superiores a 60 años y PSA basal menor de $05 \mathrm{ng} / \mathrm{ml}$ desarrollaron cáncer a 15 años, y sólo un 6,6\% de los pacientes con edad superior a 65 años y PSA basal menor de 1 $\mathrm{ng} / \mathrm{ml}$ fueron diagnosticados de cáncer a los 10 años. Por tanto, si en este grupo las determinaciones de PSA se discontinuaran en pacientes mayores de 60 años con PSA basal de $0,5 \mathrm{ng} / \mathrm{ml}$. o menor, el 100\%(IC95\%:78\%-100\%) podrían ser detectados hacia los 75 años; y si las determinaciones de PSA fueran discontinuadas a los 65 años en pacientes con un PSA basal de $1 \mathrm{ng} / \mathrm{ml}$. o menor, el 94\% de los cánceres(IC95\%:70\%$100 \%)$ podrían ser detectados hacia los 75 años. Por todo ello, podría ser oncológicamente seguro y rentable costo-efectivo no continuar los test de detección en pacientes con 65 años con niveles de PSA menores de 0,5-1 ng/ml.

¿CUÁL ES EL ALGORITMO MÁS APROPIADO?

Se han propuesto numerosos algoritmos globales ${ }^{17,20,27}$ que integran de una manera $u$ otra 
los elementos reflejadas en la literatura y que aquí hemos comentado. Hoy día no creo que se pueda asegurar cual es el mejor de todos. Creo que cada grupo deberá elegir aquel en el que más confie o se ajuste a las posibilidades de su práctica clínica diaria o lo que sería mucho mejor, aquel que se pretenda validar, ya sea con los elementos aquí vistos o incluyendo nuevas variables que puedan ser de utilidad.

Pero antes de elegir un modelo, ya sea para su uso clínico sin más o para plantear su validación, creo que se deben de hacer una serie de reflexiones a la hora de interpretar la información que nos ofrece la literatura. Y es que los resultados de cualquier algoritmo esta sujeto a múltiples influencias, que en esencia se pueden agrupar en influencias debidas a las derivadas de los distintos elementos que lo componen y a influencias de concepto, desarrollo e interpretación del modelo creado en su conjunto, y que de una manera esquemática se describen en las Tablas 2 y 3 . Pero de todos ellos me gustaría recalcar que los distintos modelos pueden haberse creado en diferentes poblaciones(general o seleccionada con criterios de riesgo de padecer cáncer de próstata, presencia se sintomas miccionales, visita al urólogo...), poblaciones que tendrán una diferente prevalencia de la enfermedad y por tanto diferentes valores postprueba (valores predictivos positivo y negativo) y en definitiva una diferente utilidad clínica, que es el concepto, no único, pero si principal que debemos de considerar. Por todo ello, se debe de ser cauto a la hora de aplicar algoritmos de cribado o de diagnostico precoz en poblaciones diferentes a las que los han originado, sobre todo cuando muchos de ellos han sido sólo propuestos en base a razonamientos teóricos o estudios retrospectivos, pero no han sido validados.

\section{CONCLUSIÓN}

Aunque hoy día existe evidencia indirecta que haría pensar en un efecto beneficioso del cribado, no hay datos que justifiquen el cribado con PSA en la población general. Así mismo, existe controversia sobre cual sería el algoritmo más apropiado en el caso de que el cribado se realice. Podemos decir, que no existe un algoritmo "definitivo" debido al carácter dinámico de
Tabla 2

Influencias técnicas en los resultados de los algoritmos de cribado y diagnósticos en el cáncer de próstata

- Tacto rectal

- Variabilidad intra e interobservador

- PSA y otras pruebas biológicas y sus índices

- Variabilidad intrasujeto

- Variabilidad inter e intraensayo

- Ecografía

- Variabilidad intra e interobservador

- Variación tecnológica

- Biopsia prostática

- Distintas técnicas

- Anatomía patológica

- Valoración diferente de las lesiones

- Tratamientos

- Distintas indicaciones

- Distintas técnicas

\section{Tabla 3}

Influencias metodológicas en los resultados de los algoritmos de cribado y diagnósticos en el cáncer de próstata

- Métodos de cribado

- Tacto rectal

- Sesgo de información

- PSA y otras pruebas biológicas

- Utilización de datos en base a serotecas

- Diferencias en manejo de serotecas

- Comparación de resultados obtenidos con técnicas no comparables

- Elaboración, presentación e interpretación de los resultados

- Muestras de población reducidas y/o no representativas y/o no comparables.

- Comparación de variables obtenidas de distintas muestras de población.

- Definición diferente de las variables resultado.

- Utilización de diferentes procedimientos estadísticos

- Sobredimensión del significado y del interés de la obtención de puntos de corte categóricos y universales.

- Utilización del concepto de "diferencia estadísticamente significativa” aislada sin valoración del concepto de "utilidad clínica" de una prueba.

- Heterogeneidad del concepto de "utilidad clínica" de una prueba.

los mismos por la rápida aparición e incorporación de nuevos avances técnicos y biológicos, pero parecería que hoy por hoy, sin dejar de incorporar el tacto rectal, se debería dar más valor a los factores personales de riesgo, y entre ellos el PSA a edades inferiores a 50 años realizando un seguimiento individualizado en función de estos factores. Los algoritmos aplicados a una población deberían de ser validados previamente en dicha población. 


\section{REFERENCIAS}

1. Allepuz C, Borque A, Rioja L. Valor de screening del antígeno específico prostático en el cáncer de próstata. Rev Clin Esp 2000;200(6): 323-324.

2. Farkas A, Scheneider D, Perrotti M, Cummings KB, Ward WS. National trends in the epidemiology of prostate cancer, 1973 to 1994: evidence for the effectiveness of prostate-specific antigen screening. Urology 1998;52:444-449.

3. Svetec D. Thompson I.M. PSA screening - current controversy. Ann Oncol 1998;9(12):1283-1288.

4. Spapen SJJC, Damhuis RAM, Kirkels WJ. Trends in the curative treatment of localized prostate cancer after the introduction of prostate-specific antigen: data from the Rotterdam Cancer Registry BJU International 2000;85:474-480.

5. Sanz Vélez JI, Allepuz Losa C, Gil Sanz MJ, et al. Diagnóstico precoz del cáncer de próstata. Análisis de 5 años. Actas Urol Esp 1997;21(9):827-834

6. Reiter RE, de Kernion JB. Epidemiology, etiology and prevention of prostate cancer. En Walsh PC, Retick $\mathrm{AB}$, Vaughan $\mathrm{AD}$ et al. Editores. Campbell's Urology $\left(8^{\text {th }}\right.$ ed.). Philadelphia(USA) Saunders Ed 2002;3003-3024.

7. Canto EI, Slawin KM. Early Management of prostate cancer: Wow to respond to an Elevated PSA. Annu Rev Med 2002;53:355-368.

8. Koning HJ, Auvinen A, Berenguer A, et al. Large-scale randomized prostate cancer screening trials: program performances in the European Randomized Screening for Prostate Cancer trial and the Prostate, Lung, Colorectal and Ovary cancer trial. Int $\mathrm{J}$ Cancer 2002;97:237-244.

9. Martín E, Luján M, Sánchez E, Herrero A, Péez A, Berenguer A. Final results of a screening campaign for prostate cancer. Eur Urol 1999 Jan;35(1):26-31.

10. Gelabert A, Arango O, Carles J, et al. Diagnóstico precoz por cribado oportunístico en cáncer de próstata. resultados de un año de protocolo. Comparación con datos históricos. Actas Urol Esp 1997;21(9):885-942.

11. Bratt O. Hereditary prostate cancer:clinical aspects. J Urol 2002;168 (3):906-913

12. Carter BS, Bova GS, Beaty TH, et al. Hereditary prostate cancer: epidemiologic and clinical features. J Urol 1993;150(3):797-802.

13. Grönberg H, Wiklund F, Damber JE. Age specific risks of familial prostate carcinoma: a basis for screening recommendations in high risk populations. Cancer 1999;86(3):477-483.

14. Bratt O, Kristoffersson U, Lundgren R, Olsson H. Familial and hereditary prostate cancer in southern Sweden. A population-based casecontrol study. Eur J Cancer 1999 Feb;35(2):272-277.

15. Carter BS, Beaty TH, Steinberg GD, Childs B, Walsh PC. Mendelian inheritance of familial prostate cancer. Proc Natl Acad Sci U S A 1992 Apr 15;89(8):3367-3371.

16. Catalona WJ, Antenor JA, Roehl KA, Moul JW. Screening for prostate cancer in high risk populations. J Urol 2002 Nov;168(5):19801983; discussion 1983-1984.

17. Dall'era MA, Evans CP. Tumors Markers. In:Carroll PR, Grossfeld GD. Prostate Cancer. Hamilton:BC Decker Inc 2002;93-112.

18. Carter HB, Pearson JD. Prostate-specific antigen testing for early diagnosis of prostate cancer: formulation of guidelines. Urology 1999 Nov;54(5):780-786.

19. Canto EI, Slawin KM. Early management of prostate cancer: how to respond to an elevated PSA? Ann Rev Med 2002;53:355-368.

20. Ross KS, Carter HB, Pearson JD, Guess HA. Comparative efficiency of prostate-specific antigen screening strategies for prostate cancer detection. JAMA 2000 Sep 20;284(11):1399-1405.

21. Schersten T, Baile MA, Asua J, Jonsson E. Detección precoz de cáncer de próstata. Síntesis y Actualización de la Evidencia. Presentación de Resultados.(Proyecto de Colaboración INAHTA). Vitoria Gasteiz: Departamento de Sanidad del Gobierno Vasco. Servicio de Evaluación de Tecnologías Sanitarias, Osteba. 1999.

22. Veltman J, Goossen T. Laguna P. Wijkstra H. de la Rosette J. New technical improvements for TRUS in the diagnosis of prostate cancer. Eur Urol 2002;1(6)(Supl 1):8-14

23. Carter HB, Partin AW. Diagnosis and staging of prostate cancer. In Walsh PC, Retik AB, Vaughan ED, Wein AJ. Editores. Campbell's Urology. 5 ed. Philadelphia: Saunders 2002;3055-3079.
24. Gretzer MB, Partin AW. PSA levels and the probability of prostate cancer on biopsy. Eur Urol 2002;1(6)(Supl 1):21-27.

25. Carvalhal GF, Smith DS, Mager DE, Ramos C, Catalona WJ. Digital rectal examination for detecting prostate cancer at prostate specific antigen levels of $4 \mathrm{ng} / \mathrm{ml}$ or less. J Urol 1999 Mar;161(3):835-839.

26. Catalona WJ, Ramos CG, Carvalhal GF, Yan Y. Lowering PSA cutoffs to enhance detection of curable prostate cancer. Urology 2000 Jun; 55(6):791-795.

27. Vis AN, Schöder FH, Van der Kwast TH. Characteristics of prostate cancer in different prostate-specific antigen ranges. In: Kurth $\mathrm{KH}$, Mickisch GH, Schröder FH, Editores. Renal, bladder, prostate and testicular cancer, an update. New York:The Parthenon Publishing Group Inc 2000;97-111.

28. Vis AN, Kranse R, Roobol M, Van der Kwast TH, Schröder FH Serendipity in detecting disease in low prostate-specific antigen ranges. BJU Int 2002 Mar;89(4):384-389.

29. Schröder FH, Van der Cruijsen-Koeter I, de Koning HJ, Vis AN, Hoedemaeker RF, Kranse R. Prostate cancer detection at low prostate specific antigen. J Urol 2000 Mar;163(3):806-812.

30. Schröder FH, Roobol-Bouts M, Vis AN, Van der Kwast T, Kranse R. Prostate-specific antigen-based early detection of prostate cancervalidation of screening without rectal examination. Urology 2001;57(1):83-90.

31. Oesterling JE, Jacobsen SJ, Chute CG, et al. Serum prostate-specific antigen in a community-based population of healthy men Establishment of age-specific reference ranges. JAMA 1993 Aug 18; 270(7):860-864.

32. Catalona WJ, Hudson MA, Scardino PT, et al. Selection of optimal prostate specific antigen cutoffs for early detection of prostate cancer: receiver operating characteristic curves. J Urol 1994 Dec;152(6 Pt 1):2037-2042.

33. Matlaga BR, Eskew LA, McCullough DL. Prostate biopsy: indications and technique. J Urol 2003 Jan;169(1):12-19.

34. Djavan B, Zlotta A, Kratzik C, et al. PSA, PSA density, PSA density of transition zone, free/total PSA ratio, and PSA velocity for early detection of prostate cancer in men with serum PSA 2.5 to $4.0 \mathrm{ng} / \mathrm{ml}$ Urology 1999 Sep;54(3):517-522.

35. Fang J, Metter EJ, Landis P, Carter HB. PSA velocity for assessing prostate cancer risk in men with PSA levels between 2.0 and 4.0 ng/ml Urology 2002 Jun;59(6):889-893.

36. Carter HB, Epstein JI, Chan DW, Fozard JL, Pearson JD. Recom mended prostate-specific antigen testing intervals for the detection of curable prostate cancer. JAMA. 1997 May 14;277(18): 1456-1460.

37. Carter HB, Epstein JI, Partin AW. Influence of age and prostate-specific antigen on the chance of curable prostate cancer among men with non palpable disease. Urology 1999 Jan;53(1):126-130.

38. Gann PH, Hennekens CH, Stampfer MJ. A prospective evaluation of plasma prostate-specific antigen for detection of prostatic cancer. JAMA 1995 Jan 25;273(4):289-294.

39. Fang J, Metter EJ, Landis P, Chan DW, Morrell CH, Carter HB, Low levels of prostate-specific antigen predict long-term risk of prostate cancer: results from the Baltimore Longitudinal Study of Aging. Urology 2001 Sep;58(3):411-416.

40. Ohori M, Swindle P. Nomograms and instruments for the initial prostate evaluation: the ability to estimate the likelihood of identifying prostate cancer. Semin Urol Oncol 2002 May;20(2):116-122.

41. Djavan B, Remzi M, Zlotta A, Seitz C, Snow P, Marberger M. Novel artificial neural network for early detection of prostate cancer. J Clin Oncol 2002 Feb 15;20(4):921-929.

42. Smith DS, Catalona WJ, Herschman JD. Longitudinal screening for prostate cancer with prostate-specific antigen. JAMA 1996 Oct 2330;276(16):1309-1315

43. Leewansangtong S, Crawford ED, Gordon SG, et al. Longtudinal Follow up from Prostate Cancer Awareness Week(PCAW): Screening Intervals. J Urol 1998;159:1977.

44. Carter HB, Landis PK, Metter EJ, Fleisher LA, Pearson JD. Prostatespecific antigen testing of older men. J Natl Cancer Inst 1999 Oct 20;91(20): 1733-1737.

Dr. C. Allepuz Losa

Hospital Universitario "Miguel Servet"

Po Isabel La Católica, 1-3 - 50009 Zaragoza

callepuz@salud.aragon.es

(Trabajo recibido el 5 de marzo 2005) 